\title{
Real time deployments of sensors and sensing patients vital parameters using secure autonomous WSN for medical applications
}

\author{
Sandip Mandal and Rama Sushil \\ Department of Information Technology, DIT University, Dehradun, India
}

\begin{abstract}
Wireless Medical Sensor Networks (WMSNs) have emerged as the most reliable technology for implementing a pervasive paradigm, facilitating doctor to patient efficiency and improving the quality of life. Sensor devices have invaded the medical domain over a recent past with wide range of capability. WSN for healthcare enable remote patient monitoring, timely exchange of health information, reminders and support thereby extending the reach of healthcare assistance anywhere, anytime. In this paper, we analyze the performance of a scalable WSN infrastructure with respect to medical applications and presents their response in scenarios which are simulated to mimic real-time behavior. All simulations have been done in MATLAB. Proposed design of an autonomic WMSN in such a way that it meets the requirements of various applications like sensed quantities, body sensor nodes autonomy, energy efficiency and reliable transmissions. The focus of the paper is on the overall network efficiency since low energy consumption, increased throughput and reliable transmission are the prerequisites for providing robust, reliable and long-lasting unhindered operations in healthcare. From the results, it shows that our autonomic WSN tries to maintain optimal amount of power for each node and also ensures that appropriate data communication occurs for all the nodes in the network.
\end{abstract}

KEY WORDS: WIRELESS MEDICAL SENSOR NETWORKS (WMSNS), WIRELESS SENSOR NETWORK (WSN), HEALTHCARE MONITORING, AUTONOMIC SYSTEM. MESH NETWORK

\section{ARTICLE INFORMATION:}

*Corresponding Author: sandy06.gcect@gmail.com Received $11^{\text {th }}$ July, 2018

Accepted after revision $23^{\text {rd }}$ Sep, 2018

BBRC Print ISSN: 0974-6455

Online ISSN: 2321-4007 CODEN: USA BBRCBA

\%.: Thomson Reuters ISI ESC / Clarivate Analytics USA and

: Crossref Indexed Journal

NAAS Journal Score 2018: 4.31 SJIF 2017: 4.196

- A Society of Science and Nature Publication, Bhopal India 2018. All rights reserved.

Online Contents Available at: http//www.bbrc.in/

DOI: $10.21786 / \mathrm{bbrc} / 11.3 / 24$ 


\section{INTRODUCTION}

Wireless medical sensor networks (WSNs) are among the most promising technologies that enable enhanced sensing capability, powerful data processing and increased communication ability from human body, within various environments and in different context. The technology offers a unique set of capabilities that produces an exciting but complex design space which is often difficult to negotiate in an application context. Deploying sensing physical environments has its own set of challenges, and can result into the system failure, thus resulting in problems that can be difficult to discover or reproduce in simulation. Sustained efforts in the area of wireless networked sensing over the last few decades have resulted in a large number of theoretical developments, substantial practical achievements. In order to bridge the gap between (on the one hand) a very large scale, randomly deployed, autonomous networks and (on the other) the actual performance of fielded systems, we need to consider deployment as an essential component in the process of developing sensor networks: a process that serve specific applications and end-user needs. Incorporating deployment into the design process reveals an entirely new and different set of considerations and requirements whose solutions require innovative thinking, strong involvement from end-user communities and multidisciplinary teams.

WMSN face particular challenges. They are deployed in hostile environments and are expected to operate efficiently within very confined technological limitations, not least of all in respect to power requirements. Maintaining the operational lifespan of the WMSN is a fundamental objective as we cannot afford to lose the medical data being transmitted in the network. While the use of intelligent techniques offer one approach, this paper will advocate the adoption autonomic principles, augmented with intelligent techniques, as the primary means by which this objective is met. The paper is structured as follows: Section I provides a brief description of WMSNs. In Section II, the notion of an autonomic WMSN is examined, with particular emphasis on the critical issues of intelligent power management, and intelligent coverage and reliable packet transmissions via message switching respectively. Section III describes the overview of the technology used. Section IV, V, VI provides the detailed motivation, architectural design and implementation of our idea respectively.

The paper specifically addresses issues of generic importance for WMSN system designers:

(i) Autonomic Behavior, (ii) Energy Efficiency, (iii) Communication Availability and Quality, (iv) Timely Data Transmission of the network designed. The main focus is the deployment and deployment evaluation.

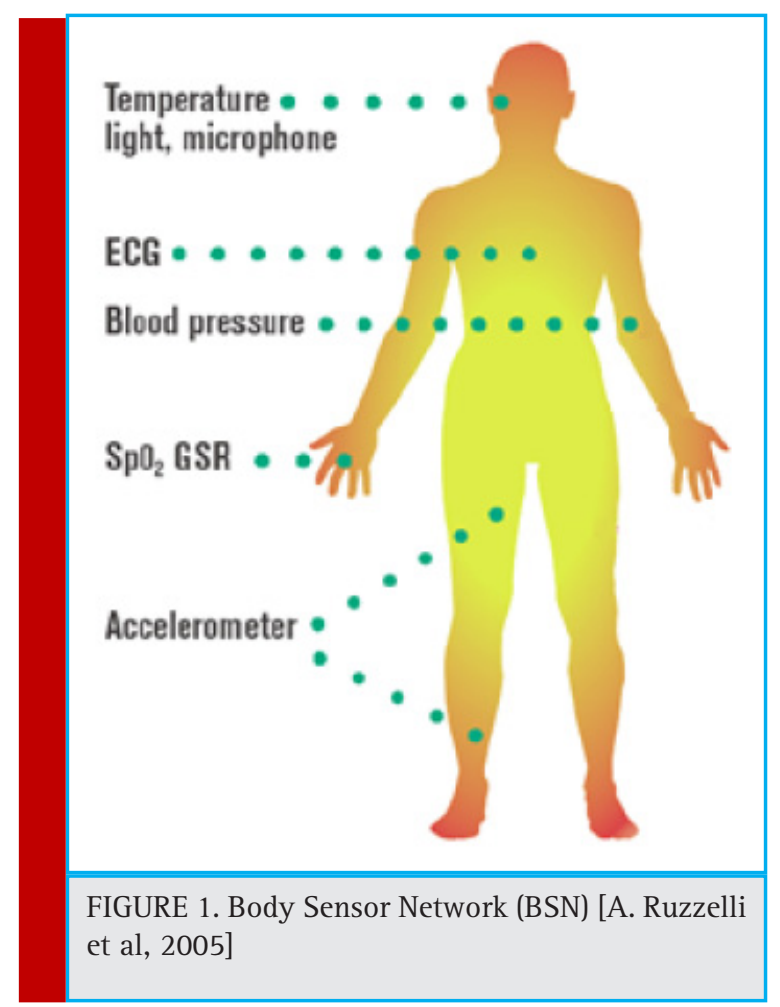

In this work, MATLAB has been used to simulate and analyze the performance of a scalable WMSN infrastructure with respect to medical applications such as pulse rate, body pressure, temperature etc. and find out how such systems respond in scenarios which are simulated to mimic real-time behavior.

\section{AUTONOMIC WSN FOR MEDICAL APPLICATIONS}

With the dramatic increase in computing devices, their increased computing capacity and complexity combined with popularity of internet, there has been a phenomenal growth in heterogeneous networks and network applications. Due to this increasing complexity, network management issues and communication protocols have reached a level beyond human ability to manage computer systems. At the highest level, the solution is to have computers manage themselves. This is achieved by providing pre knowledge to a computing element about its operation that it is capable of making informed decisions leading to its automation, self- protection and selfmanagement.

Based on the inspiration from the autonomic functioning of human central nervous system, where autonomic controls use motor neurons to send indirect messages to organs at a sub conscious level, an initiative was created by IBM towards Autonomic Computing for relieving humans from the burden of managing computer systems which is enormously growing to the extent of unmanageability. 


\section{TECHNOLOGY OVERVIEW: DEPLOYMENTS AND SENSING OF MEDICAL SENSOR NODES}

WSNs often lack robustness, are unreliable, have many elements which interoperate in complex manners, and are subject to much environmental variability. This means that it would be extremely difficult for a person to effectively administer a WSN (if access is even possible); therefore, system administrators would benefit greatly by the implementation of autonomic principle to a system (Kephart et al., 2003 and Ruzzelli et al., 2005)

To clarify the contribution that autonomic computing can bring to WMSNs, we will provide a series of scenarios in which a common problem in WMSN operation can be tackled using autonomic principles.

\section{Self Configuration}

Firstly, there is an issue of deployment. It is often assumed that the nodes forming a network cannot be perfectly positioned. Hence, a pre-programmed configuration for the network will not work. Self-configuring nodes can set up network connections, proceed to establish sensing and communication schedules and evaluate if there are any gaps in the WSN, either from a networking or sensing viewpoint. This can be well understood by studying a protocol (Ruzzelli et al, 2005) for automatically building a network out of randomly distributed nodes.

\section{Self Protection}

Secondly, there is the self-protection attribute. Sensor nodes are usually exposed to harsher environmental conditions and are thus, subjected to energy depletion and incidental damage which can lead to gradual degradation of the network as a whole. Network paths break and gaps appear in the sensing coverage of an area. A WSN needs to adapt to the changes in its topology constantly throughout its lifetime.

Therefore, WSN needs to adapt to the changes, recover from losses and be self- protected. This is achieved by renegotiation of network routes, voltage level monitoring with the sensor nodes, control over each node by base station and upon failure activating redundant nodes to replace the damaged ones.

\section{Self Healing}

Thirdly, self-healing is the ability to detect and eliminate the damage caused to the network transmissions due to the addition of some unwanted elements. (e.g., sensor transmission contaminated with some noise).

\section{Self Optimization}

Lastly, self-optimization is an important trait for WSN protocols so that maximum energy efficiency is gained from the available energy as the energy at each sensor node is limited. Given an application that uses the network, energy savings can be achieved by reducing

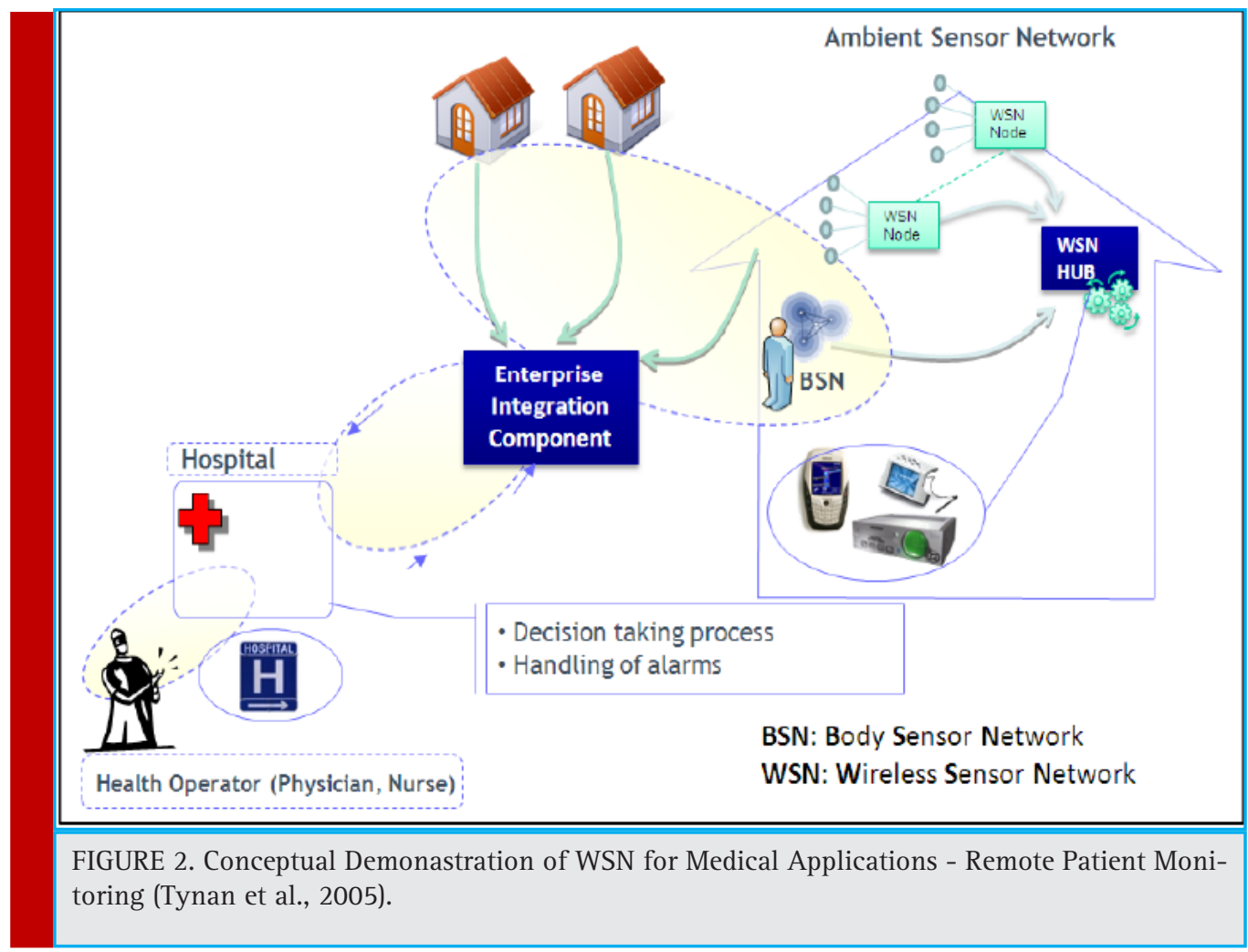


the overall performance of the network and because redundant nodes can be put into a low-power sleep mode, ready to be reactivated when the need arises. This can prove to be the best effort in achieving maximum energy efficiency. In the following subsections, we will expand on the details of the most important aspects of a WSN, namely network integrity, sensing quality and power management, and how autonomic principles aid in their optimization.

\section{Intelligent Power Management:}

Due to the deployment of WSN in a potential hazardous environment, it is of paramount importance that the network operates for as long as possible without requiring routine and frequent maintenance tasks. The most common of these tasks is battery replacement. Thus, it is vital that the nodes of the network manage their power consumption in an intelligent manner to deliver the longevity required by the network. A node's lifetime will be proportional to the amount of time it is active. Therefore limiting the nodes activity on a network wide basis will increase the lifespan of the entire network and this has proved to be one of the most effective power management techniques for WSNs [F. Ye et al, 2003]. An inactive node is termed as hibernating, a temporary state in which negligible or no power is consumed by it. Hibernating nodes are unable to report their sensed data and as such leave a blind spot in the network, where no active sensor is monitoring. The hibernation of a node must be performed in context to a network wide quality of service metric that must be maintained by the active nodes. For power management, this is typically coverage. Each sensor is associated with a sensing radius within which it can sense and outside which it is dormant. Some techniques use an inverse distance relationship between the sensor and its ability to sense but there is still a limit to its sensing capability. An area is deemed covered if the union of all the active sensors sensing discs includes every point within the sensed area. This is the constraint under which nodes may be hibernated, thus managing the networks power consumption intelligently while maintaining a quality of service with regard to its surveillance density. Algorithms based on this principle include CCP [H. Zhang et al, 2005] and OGDC [F. Ye et al, 2003]. These techniques operate on an optimization of the problem, ensuring that the intersection of two sensing discs is covered by another sensor and that every intersection point of the sensors discs within the boundary area are also covered by another sensor. Using this property the algorithms manage the nodes activity and limit the power consumption by the network. Another technique based on the standard coverage maintenance is interpolation, and more specifically interpolation error [R.Tynan et al, 2005]. For a given sensor network meant for sensing tem- perature for example, there will be a temperature distribution function across the area of the network. Sensors of the network are responsible for sampling this function at discrete locations within the area and report their readings to a base station. Interpolation is a mathematical technique for approximation of function values between known values of that function. The known points of the temperature function are at the locations of the sensors within the context of sensor networks. Therefore, interpolation could be used to approximate the temperature between the sensors. A set of sensors used to approximate another sensor's temperature reading and then comparing it to the actual reading at the sensor's location is the interpolation error. Hibernating sensors whose interpolation error is less than a particular defined application threshold, then the node is hibernated, thus conserving further amounts of energy. This interpolation technique is the first coverage technique that includes neighboring node locations as well as neighboring node readings and can deliver greater savings.

\section{Intelligent Routing:}

Forwarding of packets from a source to a destination is the fundamental feature of multi-hop networks. In such networks, a node should be capable of identifying the best node to pass on the packet so that it reaches the destination in time. The easiest way to do it would be to flood the entire network; however, this is an unfeasible approach due to the energy consumption and packet overhead that the flooding would cause. An efficient routing poses great challenges such as which node to forward the packet among all neighbouring nodes and the trade-off between lightweight (but with high delay) reactive on-demand type routing and low latency (but computational heavy) of proactive type routing. Proactive protocols try to keep an accurate snapshot of the networks working status and maintain full knowledge of the system; hence they are suitable for high computational capability devices but the low processing capability of sensors prevents them possessing a full proactive routing protocol. On the contrary, on-demand routing protocols exempt from maintain a routing table all the time but only build it when a packet needs to be relayed to a certain destination. This is not an easy task, especially when the destination is unknown, and the situation is exacerbated when sensor node energy constraints do not permit continuous network flooding of packets. A secondary issue concerns global node addressing, which causes to prevent functioning of nodes in two different parts of the networks from having the same ID; an undesirable situation which can lead to incorrect routing for certain destinations. Apparently, this is not a trivial problem, particularly when dealing with large scale sensor networks that may comprise thousands of units. The 
literature survey reveals a profusion of routing protocols, all tailored to wireless sensor networks. However, before delving into the relevant approach proposed, it is important to understand the necessary perquisites for a routing algorithm to be apt for sensor networks:

1. Self-organization, as the expended nodes might or might not be substituted with new ones; hence it is important for the network to be prepared in advance for sudden changes of devices and possibly their location.

2. Flexibility, external factors such as temporary or permanent obstacles, might cause the network topology to change continuously due to which neighboring nodes may be disconnected. This can result in the need for the identification of a new routing path.

3. Scalability, a sensor network consists of a certain number of nodes that may span from single figures to tens of thousands of devices.

4. Lightweight, due to limited processing capability single devices may be prevented from having a high computational load.

5. Energy-efficiency, nodes run on batteries and need may arise to scavenge energy from the environment. Furthermore, nodes might be deployed in remote areas where it is impractical to recharge them.

6. Loop-free, the routing algorithm should ensure that the packets are not routed endlessly around the network without finding the correct destination.

7. Reliability, the protocol should guarantee a high percentage of correctly routed packets to the destination.

8. Tolerable latency, some application need to receive the packets requested within a certain deadline. Thus, it would be preferable to have a protocol that can autonomously trade off energy savings for packet delay as per the application needs.

All the above characteristics imply the need for an Intelligent Routing protocol for autonomic wireless sensor networks (Ruzzelli et al., 2005).

\section{Intelligent Coverage:}

One of the most important aspects of a sensor network is to have satisfactory coverage i.e. the ability to provide sensory data of sufficient quality to the application which is using it, At the highest level, what concerns the most is that the sampling frequency, both in spatial and temporal terms, is high enough so that the phenomena of interest can be observed in sufficient detail. However, considering WSNs, we must also be aware of the energy cost of any actions. As suggested earlier, it is necessary to balance the level of detail the network is providing to the client against the rate at which energy is being consumed while gathering the data. Clearly, it is preferable to tune the network automatically, rather than doing it manually. Autonomic computing helps in intelligent reasoning about the coverage in the network. Various methods can be used to change the quality of the sensory data the network produces. The simplest among them is to reduce the rate at which the sensors sample the environment. This increases the time period the sensor nodes can spend in a low-power sleep state, and is relatively easy to coordinate. A more complex mechanism is to identify nodes that are not required due to a high density of nodes in a particular area $[\mathrm{H}$. Zhang et al, 2005]. This needs coordination between the nodes on a local level to decide which ones should sleep and which ones should enter an active state. If the range of the sensors is variable, then varying this parameter can be put to use is one more way to fine tune the power/sensing relationship [D. Marsh et al, 2005]. When flooded with pre-calculated knowledge of the relationship between deployment densities, coverage levels and sensor ranges, a node can alter these variables while avoiding combinations that would have an adverse effect on the network as a whole. Finally, mobile nodes have the capability to reposition themselves as conditions dictate [K. H. Low et al, 2006]. In a mixed network of fixed and mobile sensors, the preservation of coverage in areas where too many static sensors have become depleted or damaged (often considered a failure condition for WSNs) is facilitated by this. In mobile networks, nodes effectively occupy two or more spatial locations once they switch positions faster than the requisite temporal sampling rate, thus restricting down on the number of nodes needed. A special case occurs when an inherently static node with independent decision making is attached to a mobile object, for instance a vehicle or person. In this instance coverage becomes a probabilistic problem, since no actions on the WSN's part can influence where the nodes will end up. In all types of coverage that a WSN requires, there exist tradeoffs. It is often possible to reduce communication rates for an increase in processing time. When mobile nodes are equipped with an appropriate sensing modality, sensing can be utilized to observe other nodes, rather than using the radio to exchange positions (Low et al., 2006). Thus, depending on the relative costs of sensing, processing and radio communication, a sensor network can dynamically choose to favour one method over the other if it leads to significant energy savings. By intelligently choosing the optimal alternative, an autonomic sensor network can achieve energy savings to a level beyond what could be expected from a standard WSN. 


\section{MOTIVATION AND PROPOSED SYSTEM MODEL}

Modern wireless sensor technologies enable powerful data processing, enhanced sensing capability, and increased communication ability from human body, in different context within various environments. With increased prevalence of chronic diseases all over the world, there is constant pressure on healthcare system to find ways to deliver reliable healthcare solutions that can provide same service at affordable cost and the services that do not need intensive enrolment of medical staff. In critical emergency response scenarios, designing a medical sensor network that can deliver suitable functionality (e.g. energy efficiency, throughput, sensor data transmission rate) to meet the evolving patient, provider, and workflow needs is a critical challenge. The use of wireless technologies in medical environment is bringing major advancement to the existing healthcare services. However, these have several key research challenges such as various types of network communication infrastructure, fault-tolerance, data integrity, low-power consumption, transmission delay, node failure, etc. Reliability is one of the most important factors in a successful healthcare system. To ensure this factor, system designers have to care about adaptation of nodes when its location, connection and link quality is changed. Different network communications infrastructure should be used in appropriate situation. For example, with highrisk patients, higher QoS services should be used. The integrity of distributed data system and fault-tolerance should be given a proper consideration also. Every device operates differently at different times, especially sensor devices. A node in a network can be fail at anytime for number of reason including battery exhaustion, human-related issues or natural issues. Ensuring a seamless service during life time of the network could be a big challenge. How to manage the transmission delay of various types of communications in the network is an undoubted challenge. With the system using WMSN, data must undergo reduced hop counts before it reaches the sink. In addition, these hops are sometime located in very critical conditions, such as areas bearing interference of radio waves or magnetic field. As a result, various transmission delays can occur and thus, require extra effort of system designer to synchronize the whole network.

While deploying a WMSN all sensor nodes are communicating to the central server i.e. hub through a wireless protocol. If the transmitted packets containing patient data are not routed properly due to routing congestion, there is likelihood that the packets containing patient's vital information will reach late or not reach the desired destination at all. This leads to packet delay which is strictly undesirable in WMSN as diagno- sis delayed is as good as diagnosis not done. Also the problem of packet delay is due to the coverage area as medical sensor networks consists of millions and billions of sensor nodes deployed within a particular range. Suppose the range is $\mathrm{x}$ metres and the nodes in this particular range become so dense that communication is effected to an extent that leads to packet delay. To counter attack this situation our priority is to reduce the effective area of the network by finding a common balance between the number of nodes in a given region and the number of packets they will transmit. Further, the sensor nodes deployed in the network are continuously communicating altogether at the same time. Thus, there becomes a possibility that some of the nodes become vulnerable and might get hacked.

We are introducing a dynamic medical sensor network architectural paradigm, where any of the routing protocol and security algorithms have the ability to tune themselves to suit the usage scenario. The sensor network can be statically configured prior to deployment and dynamically reconfigured during operation.

\section{VERACIOUS WIRELESS MEDICAL SENSOR ARCHITECTONICS -VWMSA}

As a solution for listed problems we have proposed and created VWMSA - Veracious Wireless Medical Sensor Architectonics The aim of VWMSA is to serve as a very simple but robust wireless sensor architecture that can help in designing a reliable, scalable and fault tolerant topology meant for WSN. An efficient architecture should be capable of offering:

- High throughput

- Small overhead

- Low or manageable latency

- Optimal power consumption

- Excellent ultra-low power performance in continuous reliable transmission of data, and large data traffic.

In order to accomplish all the above goals we create a WSN topology - VWMSA which is intended to provide energy efficiency communication, both for reliable transmission of data, and for data streaming. It incorporates autonomic behavior which is very much desirable in modern WSN also a method of packet switching known as message switching. Thus, it contains proprietary communication solution methods which are ideal for medical applications as we can't afford to lose any data being transferred in the network.

VWMSA shows an autonomic behavior where the central server can place the nodes into active and sleep mode but once the nodes are active, they can take their own decisions in real time. Also the nodes can com- 


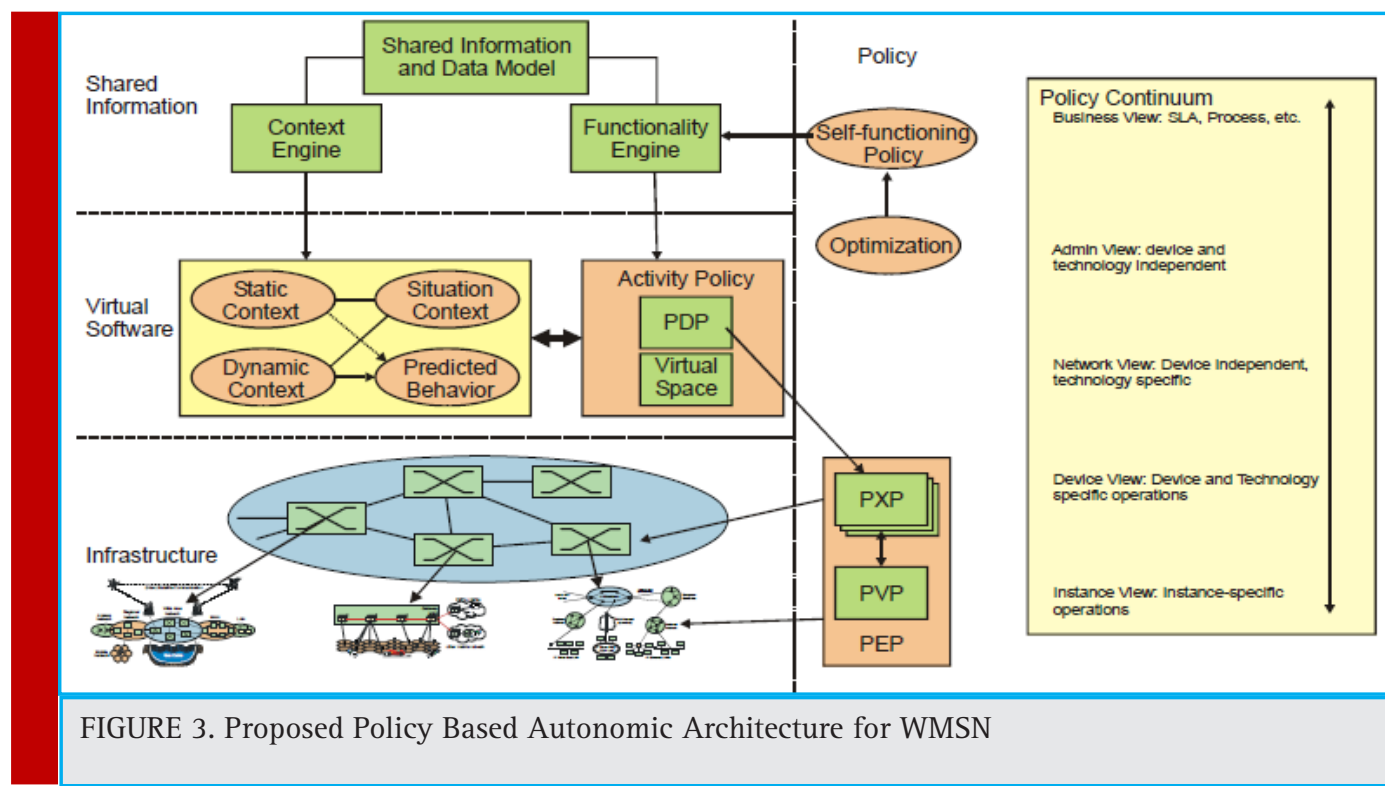

municate with each other in real time through a packet switching technique called message switching where the packets are routed from the source node to the destination node, in their entirety, one hop at a time.

In message switching [Cohler et al, 1967], the source and destination nodes are not involved in direct communications. Instead, the intermediary node (central server) is responsible for transferring the data from one node to the other by the process of activation/deactivation of the source node. It is only after the source node is set up into transmission mode i.e. when it receives the clearance or acknowledgement from the destination node, packets are transmitted in just one go preventing malicious packet to enter the network. As shown in Figure 4 data packet P1 is transmitted from $\mathrm{A}-\mathrm{C}$ and data packet $\mathrm{P} 2$ is transmitted from D - B. Data P1 follows the route A $\rightarrow \mathrm{I} \rightarrow \mathrm{II} \rightarrow \mathrm{III} \rightarrow \mathrm{C}$ and $\mathrm{P} 2$ follows the route $\mathrm{D} \rightarrow \mathrm{IV} \rightarrow \mathrm{II} \rightarrow \mathrm{B}$ depending upon the availability of the free path at that particular moment.

\section{IMPLEMENTATION AND PERFORMANCE ANALYSIS OF WMSN}

The purpose of this prototype is to assess the performance of a Wireless Sensor Network primarily targeted for medical applications. Our primary concern is to verify that all the nodes are always able to communicate their data to the main server and that network performance is fairly uniform over the entire duration of the operation (defined by simulation time). Our approach is to create coherent wireless sensor network architecture with arbitrary number of nodes assumed to be scattered in a 2-dimensional space. The next step is to define a proper topology that can ensure that the data is always safely transmitted and shared within the network. For this we create an adaptive segmentation of regions each having a finite number of nodes i.e. continuous changes with each time stamp to avoid security vulnerabilities. During this region wise segmentation, all the nodes will be used

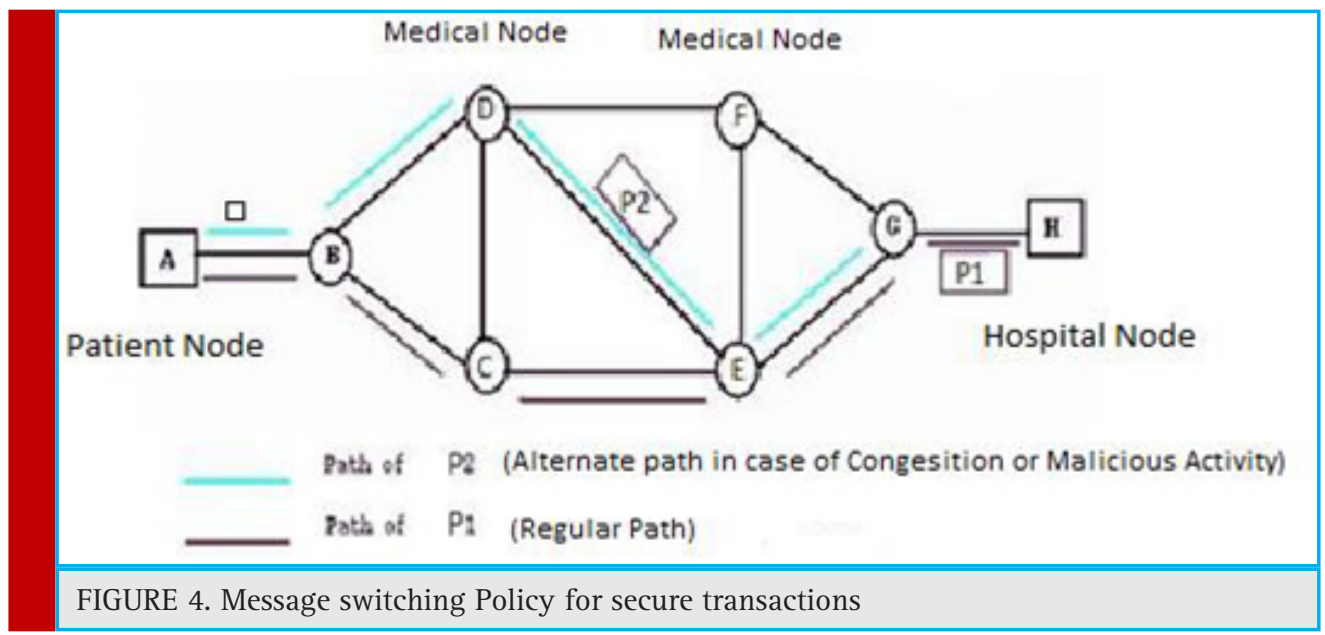




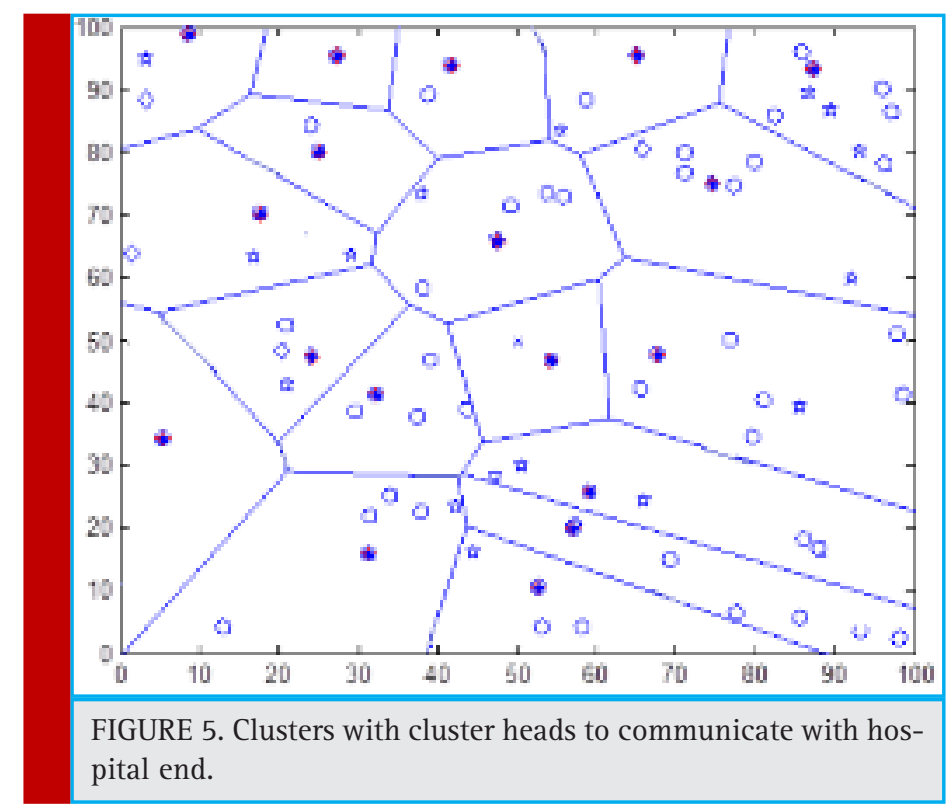

to communicate with each other through packet transmission. It is assumed that only one packet is transmitted every time stamp. Fig. 5 shows the clustering of the various sensor nodes representing various hospital data.
The region wise segmentation is performed based on the real-time data being transmitted and how important the data is. These regions are guaranteed to have reliable operation since network traffic is optimal. We intercon-

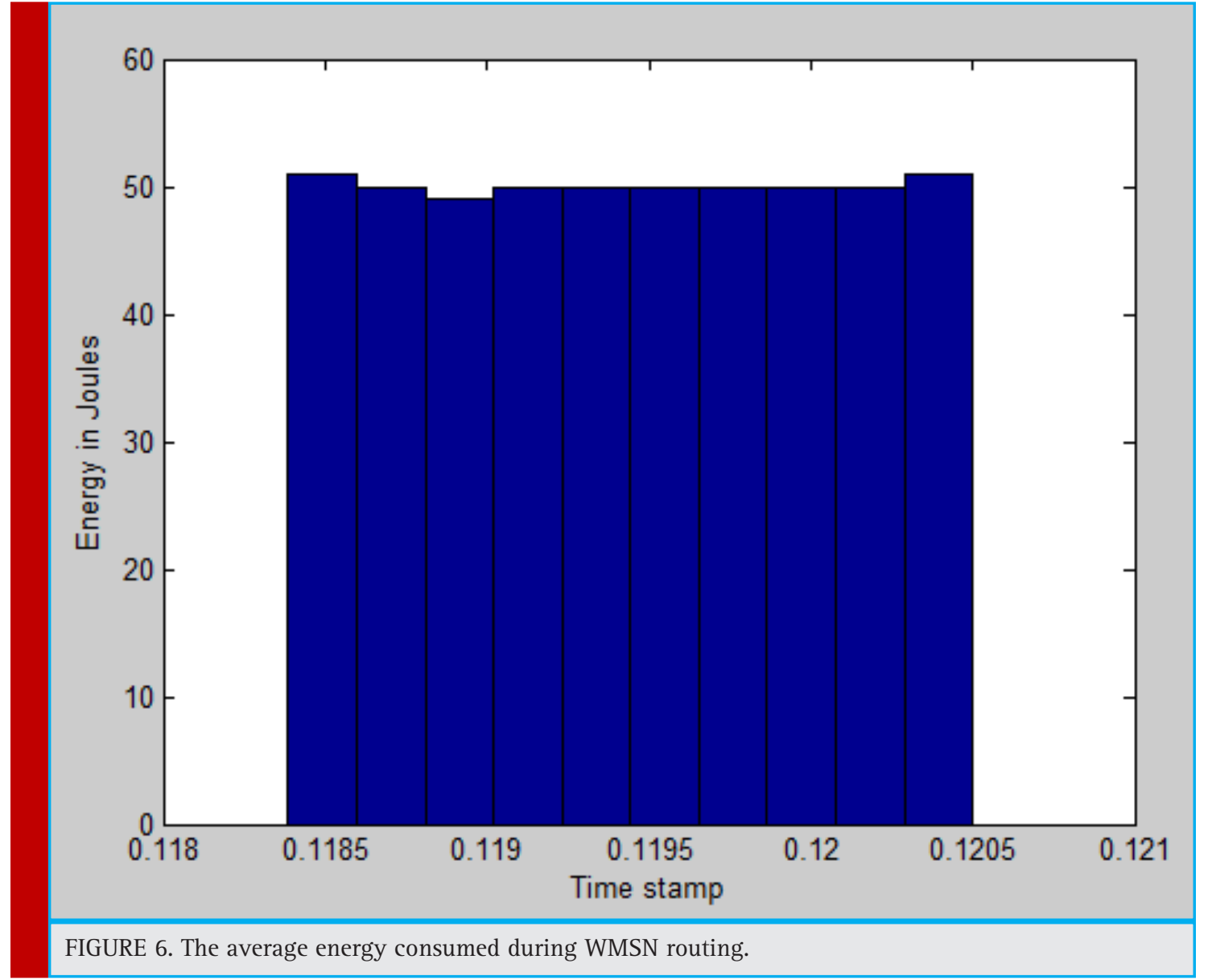


nect these regions to form a global network thereby assuring optimal performance of the entire WMSN by overall reducing the effective area of the network.

Once the operation of the WMSN is simulated (using event driven simulation) we collect several important parametric data and use it as benchmarks for assessment of the WMSN model created and check its reliability in terms of energy efficiency, clustering density and throughput analysis. The efficiency of the proposed approach is based on the simulation studies that have been performed using MATLAB ${ }^{\oplus}$.

\section{Energy Efficiency}

The average power consumption of the sensor nodes and the network lifetime is related to the work-idle-work intervals. Evidently, the longer the intervals lower the power consumption. The adaptive working mode can be applied in most of scenarios in WMSN. For increasing the network lifetime, nodes are configured to "Sleep" or power saving mode in which the sensor nodes could turn off most of the modules, greatly reducing quiescent power consumption under idle state. In contrast, dataintensive nodes are configured to the "Continuous" or "Standby" power saving mode in which the sensor nodes work continuously, for data collecting and transmission. When the communication process is done we have to find the measure of the network performance where the communication is done satisfactorily and all the nodes are performing well. This depends on the amount of energy being consumed in the network and it should be uniform across all the nodes for the communication to take place reliably.

\section{Cluster Density}

Hierarchical based clustering seems to be ideal for WMSNs. It ensures energy efficient routing by forming local clusters and transmitting the information to the gateway nodes based on the events incurred in an adjacent area followed by data aggregation by means of the gateway nodes. The clustering and re-clustering designs are simple and reliable [Sung-Hwa Hong et al, 2013].

Cluster head selection is performed in a greedy manner via the local exchange of node energy states. Each cluster head determines when to abandon its role and become a cluster member, depending only on its own energy state.

These local interactions and local decisions regarding clustering and re-clustering increase scalability and reduce control overhead at the cost of reduced optimality. The clustered structure is not for the routing purposes.

Routing information is managed independently from cluster structure. A cluster member transmits packets only to its local cluster head, but a local cluster head can transmit packets to any nodes that can route the packets to the central server or the hub node which is where the

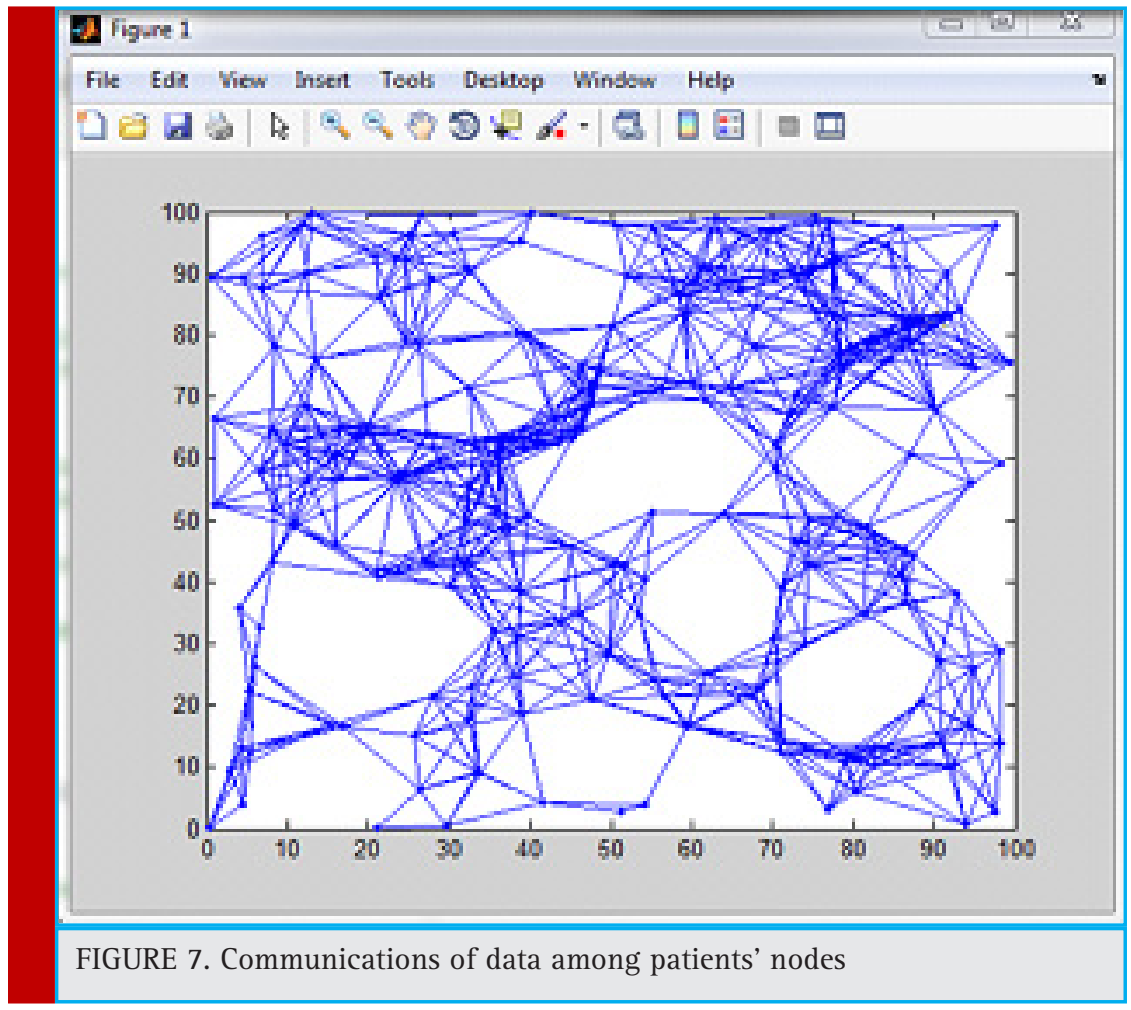




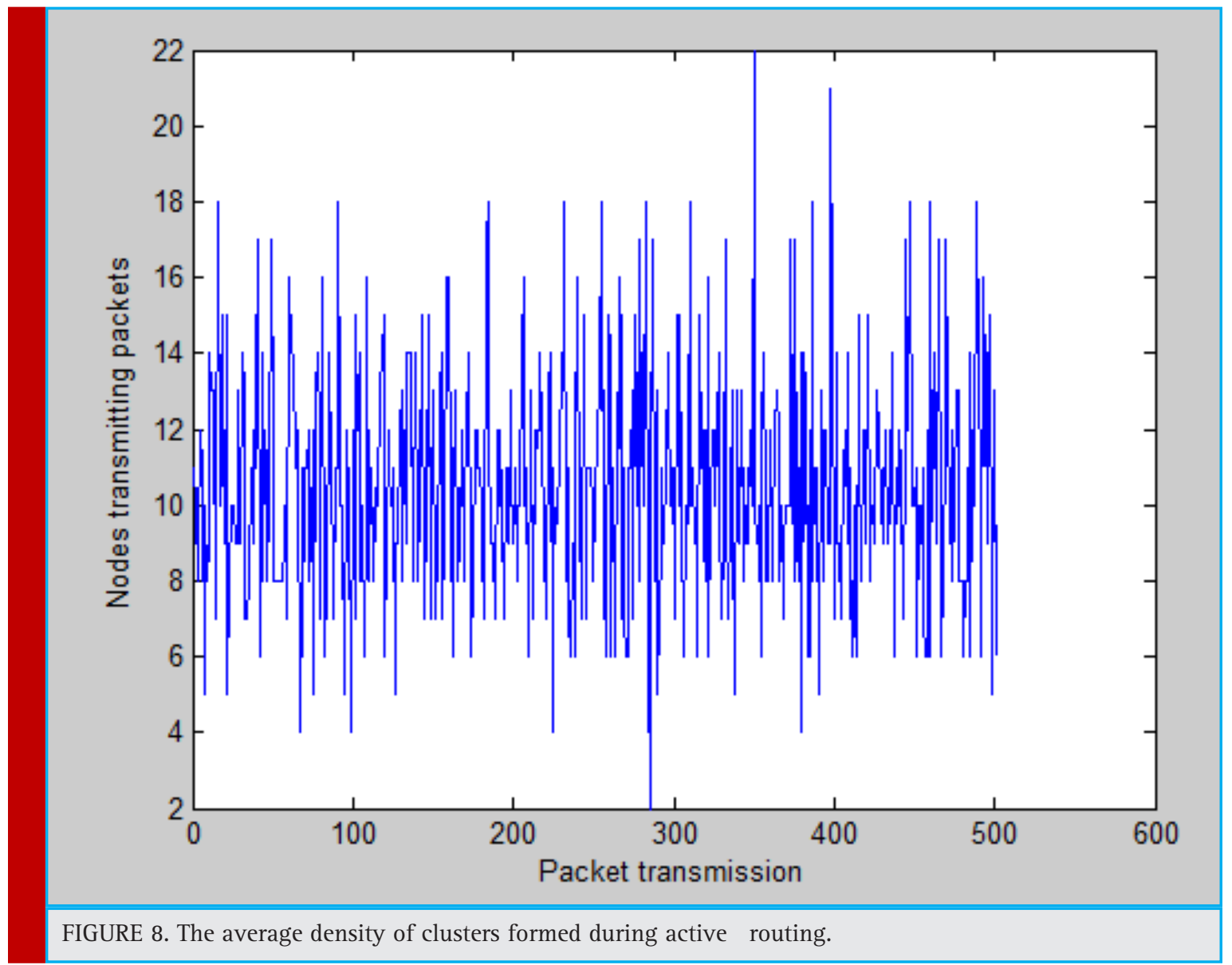

doctor has an access to the patient's data. Fig.7 shows the average density of clusters being formed while active routing is being performed.

This has similarity to a random variable which is not surprising as multiple routes can be used in the WMSN for delivery of the packets.

\section{Throughput}

The percentage of total packets received successfully, is known as throughput of the network or packet delivery ratio. It is expressed as:

In Fig.9, almost all nodes show uniform packet transmissions since, they send relatively greater number of packets in the network. The plot shows the average throughput of the sensor nodes in a given region specifying how the data packets are uniformly distributed with respect to the time.

\section{CONCLUSIONS AND FUTURE WORK}

Wireless sensor networks for healthcare certainly have developed to a stage where their usefulness in health- care application is undoubted, but the technology is still at an early stage of development. Problems that need to be solved in order to facilitate the use of the sensor networks in medical environment are lack of standardization and therefore low interoperability. In this paper, a relaying energy-efficient heterogeneous WMSN architecture for patient monitoring is proposed. This architecture compatibly incorporates various routing protocols and security algorithms that define the minimum energy parameters for the sensors to avoid damage to the human lives. Main focus lies on the autonomic architectural design of WSN for medical applications. The results are satisfactory and clearly show that in terms of the network lifetime and stability, our proposal is new, better and efficient when considering the energy efficiency, reliable packet transmissions, throughput and latency in the network as compared with the various existing routing techniques.

In future we intend to implement the presented scenario on human body with physical sensor nodes and performing comparison it with other existing mechanism. 


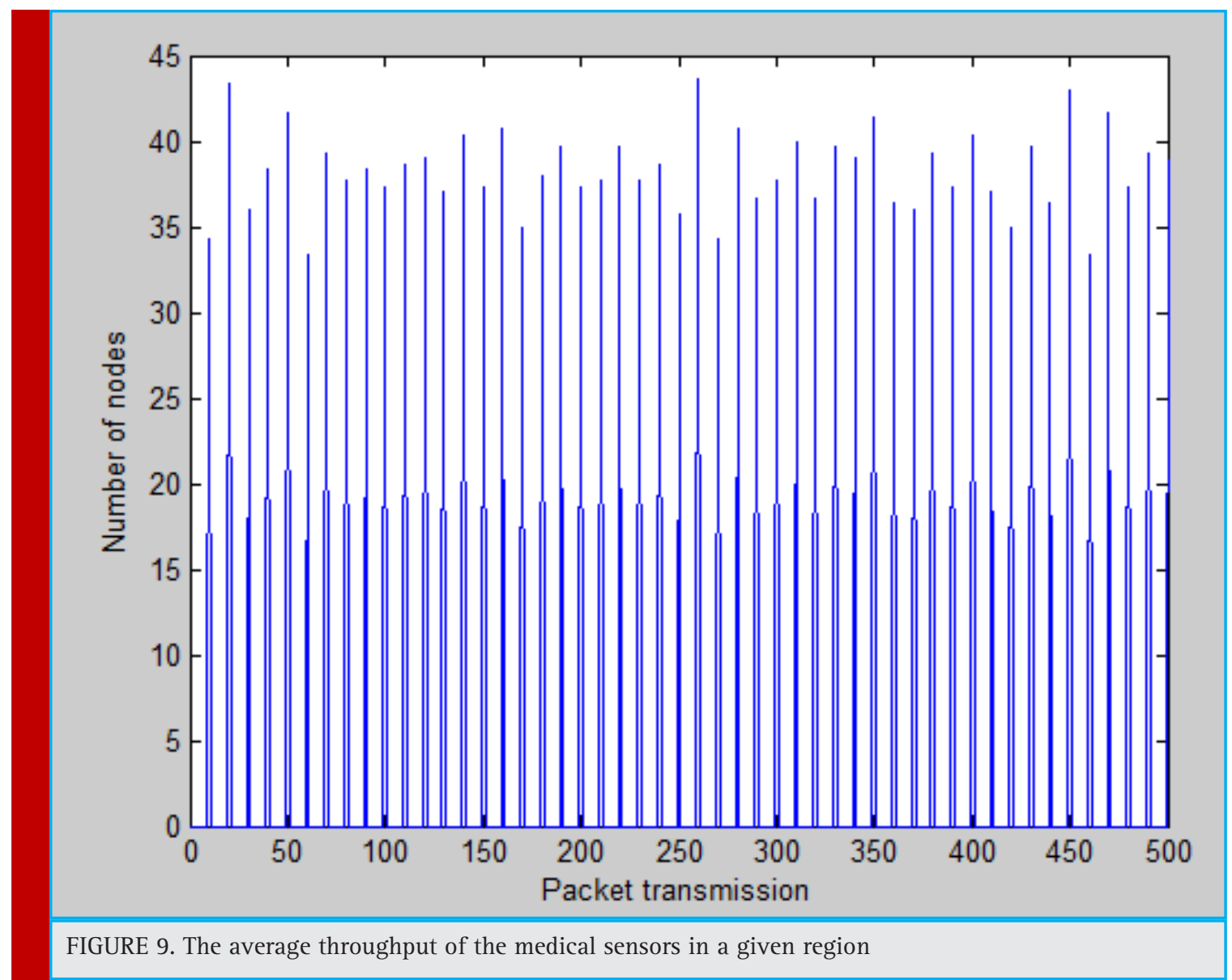

\section{REFERENCES}

IBM (2005) An architectural blueprint for autonomic computing Autonomic Computing White Paper ${ }^{\circ}$ Copyright IBM Corporation 2005.

Demirkol, C. E., Alagoz (2006) Mac protocols for wireless sensor networks IEEE Communication Magazine, Vol.06 Isuue 1, pp 115-121.

Stankovic J.A, Cao Q, Doan T, Fang L., He Z, Kiran R (2005) Wireless Sensor Networks for In-Home Healthcare: Potential and Challenges in Proc. of High Confidence Medical Node Software and Systems (HCMDSS) Workshop, Philadelphia.

Haenggi M, Andrews F, Baccelli F, Dousse 0, and Franceschetti 0 (2009) Stochastic geometry and random graphs for the analysis and design of wireless networks IEEE J. Sel. Areas Comm., Vol. 27 Issue 3, pp 1029-1046.

Kar S and Moura J (2008) Sensor networks with random links: Topology design for distributed consensus ,IEEE Trans. Signal Processing, Vol. 56, pp 3315- 3326.

Hung, K.; Zhang, Y.T. (2002) Usage of Bluetooth in wireless sensors for telehealthcare, Engineering in Medicine and Biology, 2002, 24 ${ }^{\text {th }}$ Annual Conference of the IEEE. Vol. 3, pp 1881-1882.

Borromeo S., Rodriguez-Sanchez C., Machado F., HernandezTamames J.A. (2007), A Reconfigurable, Wearable, Wireless
ECG System, Engineering in Medicine and Biology Society $29^{\text {th }}$ Annual International Conf. of the IEEE, pp 16591662.

Kephart J.0. and Chess D.M. (2003), The vision of autonomic computing, IEEE Computer, Vol. 36-1, pp 41-50.

Ruzzelli, O'Hare G.M.P, O'Grady M.J., Tynan R. (2005), Adaptive scheduling in wireless sensor networks 2nd IFIP International Workshop on Autonomic Communication, Vouliagmeni, Athens, Greece.

Qi H, Kuruganti P.T., Xu Y. (2002), The development of localized algorithms in wireless sensor networks ,Sensors, Vol. 2, pp 286-293.

F. Ye, G. Zhong, J. Cheng, S. Lu, L. Zhang (2003), “ PEAS: A Robust Energy Conserving Protocol for Long-lived Sensor Networks", Proceedings of the 23rd International Conference on Distributed Computing Systems, IEEE Computer Society, pp 0-7695-1920-2.

H. Zhang, J. C. Hou(2005), "Maintaining Sensing Coverage and Connectivity in Large Sensor Networks", Ad Hoc \&t Sensor Wireless Networks, Vol. 1, pp 89-124.

R.Tynan, G.M.P. O'Hare, D.Marsh, D, O'Kane (2005), “Interpolation for Wireless Sensor Network Coverage", EmNetS-II:The Second IEEE Workshop on Embedded Networked Sensors, Sydney, Australia. 
Marsh D., Tynan R, O'Hare G. M. P., Ruzzelli A. (2005), The effects of deployment irregularity on coverage in wireless sensor networks, 2nd International Conference on Intelligent Sensors, Sensor Networks and Information Processing (ISSNIP 2005), Melbourne, Australia.

Low K. H., Leow W. K., Ang M. H., (2006), Autonomic mobile sensor network with self-coordinated task allocation and execution, IEEE Trans. on Systems, Man and Cybernetics - Part C: Applications and Reviews, Vol. 36-3, pp 315-327.

Cohler, Edmund U., Rubinstein, Harvey (1967), A Multicomputer Message Switching Data Processing System, IEEE Trans. on Communication Technology, - Part C: Applications and Reviews, Vol. 15, pp 314-321.
Sung-Hwa Hong, Jeong-Min Park,Joon-Min Gil(2013), Performance Evaluation of a Simple Cluster-Based Aggregation and Routing in Wireless Sensor Networks, International Journal of Distributed Sensor Networks, Vol. 2013, Article ID 501594, pp 612-621.

Mandal S, Sushil R (2018) Enhanced Energy-Balanced Lifetime Enhancing Clustering for WSN (EEBLEC), International Journal of Applied Engineering Research ,Vol 13, Issue 16, pp. 1291112916 .

Mandal S, Sushil R (2018) Energy Saving Dynamic Clustering Protocol for Wireless Sensor Network, International Journal of Engineering Applied and Management Sciences Paradigms, Vol 53 , Issue 3, pp. 25-28. 